\title{
Interleukin-37 is increased in peripheral blood mononuclear cells of coronary heart disease patients and inhibits the inflammatory reaction
}

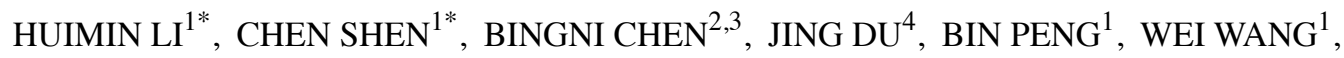 \\ FANWU CHI $^{1}$, XIAOQIANG DONG ${ }^{1}$, ZHONG HUANG $^{2,3}$ and CHAO YANG ${ }^{1}$ \\ ${ }^{1}$ Department of Cardiac Surgery, Shenzhen People's Hospital, Second Clinical Medical College of Jinan University, \\ Shenzhen, Guangdong 518020; ${ }^{2}$ Department of Pathology, Shenzhen University School of Medicine; \\ ${ }^{3}$ Department of Immunology, Shenzhen City Shenzhen University Immunodiagnostic Technology Platforms, \\ Shenzhen, Guangdong 518060; ${ }^{4}$ Department of Laboratory Medicine, Peking University \\ Shenzhen Hospital, Shenzhen, Guangdong 518036, P.R. China
}

Received June 10, 2019; Accepted October 16, 2019

DOI: $10.3892 / \mathrm{mmr} .2019 .10805$

\begin{abstract}
It has been universally acknowledged that interleukin-37 (IL-37) has an immunosuppressive effect on various inflammatory disorders. However, whether IL-37 participates in the acute inflammation associated with coronary heart disease (CHD) has not yet been clarified. In the present study, the association between the serum levels of IL-37 and the clinical indexes of CHD were analysed. In addition, the
\end{abstract}

Correspondence to: Dr Chao Yang, Department of Cardiac Surgery, Shenzhen People's Hospital, Second Clinical Medical College of Jinan University, 1017 Dongmen North Street, Shenzhen, Guangdong 518020, P.R. China

E-mail: ychaosz@126.com

Dr Zhong Huang, Department of Immunology, Shenzhen City Shenzhen University Immunodiagnostic Technology Platforms, W2-A Block, 27 Gaoxin South Forth Road, Yuehai Street, Shenzhen, Guangdong 518060, P.R. China

E-mail: zhuang809@126.com

*Contributed equally

Abbreviations: IL-37, interleukin-37; CHD, coronary heart disease; PBMCs, peripheral blood mononuclear cells; AMI, acute myocardial infarction; UAP, unstable angina pectoris; SAP, stable angina pectoris; HCs, healthy controls; RT-PCR, real-time polymerase chain reaction; ELISA, enzyme-linked immunosorbent assay; cTnT, cardiac troponin T; NT-proBNP, amino-N-terminal pro-plasma brain natriuretic peptide; LVEF, left ventricular ejection fraction; $\mathrm{CHOL}$, cholesterol; TG, triglyceride; LDL, low density lipoprotein; HDL, high density lipoprotein; SLE, systemic lupus erythematosus; GD, Graves' Disease; AS, ankylosing spondylitis; PDGF, platelet-derived growth factor; FGF, fibroblast growth factor; SMC, smooth muscle cells; NF, nuclear factor; AP, activator protein

Key words: interleukin-37, coronary heart disease, atherosclerosis, inflammation, pro-inflammatory cytokines anti-inflammatory effects of IL-37 on peripheral blood mononuclear cells (PBMCs) were studied in CHD patients. PBMCs from 46 healthy controls (HCs) and 92 CHD patients were cultured in vitro and stimulated using the recombinant IL-37 protein. The protein levels, as well as the mRNA expression of inflammatory cytokines (TNF- $\alpha$, IL-1 $\beta$, IL-6, and IL-17) were analysed by enzyme-linked immunosorbent assay (ELISA) and real-time polymerase chain reaction (RT-PCR). Spearman's correlation test was performed to examine the association between the serum level of IL-37 and the levels of pro-inflammatory cytokines, certain clinical indexes, and disease activity during CHD. Compared to the HCs, the CHD patients, especially those with acute myocardial infarction, exhibited higher levels of IL-37 in their PBMCs and sera. Serum levels of IL-37 were associated with the levels of IL-17, IL-6, and TNF- $\alpha$, and clinical indexes such as the left ventricular ejection fraction (LVEF), amino-N-terminal pro-plasma brain natriuretic peptide (NT-proBNP) levels, and cardiac troponin $\mathrm{T}$ (cTnT) levels in CHD patients. Compared to the $\mathrm{HC}$ group, the production of inflammatory cytokines such as IL-17, IL-6, TNF- $\alpha$, and IL- $1 \beta$ increased in the PBMCs of CHD patients and significantly decreased after the stimulation of the cells with recombinant IL-37. The IL-37 levels in CHD patients were high, and were correlated with the levels of CHD-related pro-inflammatory cytokines and disease activity. Notably, the expression of CHD-related pro-inflammatory cytokines in the PBMCs of CHD patients decreased following the stimulation of the cells with recombinant IL-37, indicating that IL-37 exerts anti-inflammatory effects during CHD.

\section{Introduction}

Coronary heart disease (CHD) refers to a group of diseases involving acute myocardial infarction (AMI), stable angina pectoris (SAP), and unstable angina pectoris (UAP). CHD may trigger heart attacks, heart failure, arrhythmia, and even sudden cardiac death (1). Accumulating evidence has 
underlined that inflammatory responses are involved in the overall pathological process of CHD, including the development of atherosclerosis, thrombus formation, and the ischemia of cardiomyocytes during heart attack $(2,3)$.

Recent studies have demonstrated that multiple inflammatory cells participate in the nosogenesis of CHD, especially peripheral blood mononuclear cells (PBMCs) (4-8). In addition, numerous inflammatory cytokines lead to the inflammatory response associated with CHD, including IL-17, IL-6, TNF- $\alpha$, and IL-1 $\beta$ (9-19). However, the specific mechanism underlying the inflammatory activity in CHD has not yet been defined. A previous study undertaken by our research group revealed that IL-37 participates in peripheral tolerance during systemic lupus erythematous (SLE) through the regulation of the inflammatory cytokines IL-6 and TNF- $\alpha$ (20). Moreover, Boraschi et al revealed that IL-37 is highly expressed in human atherosclerotic plaque foam cells, indicating that IL-37 may be involved in the nosogenesis and progression of CHD (21).

IL-37, a human cytokine that has recently received an increasing amount of attention, has been revealed to have a broad protective effect against diseases caused by inflammatory responses $(21,22)$. It has been confirmed to be involved in many chronic inflammatory diseases such as SLE and plays a role as an anti-inflammatory cytokine in these diseases by downregulating the expression of pro-inflammatory cytokines (20). As for coronary heart diseases, on the one hand, it was reported that IL-37 was increased in patients with arterial calcification, a predictor of coronary heart events (23). On the other hand, in an animal experiment, treatment with IL-37 in old endotoxemic mice could improve cardiac function via suppression of myocardial inflammation (24). However, it is unclear how the anti-inflammatory properties of IL-37 occur during cardiovascular diseases, especially CHD.

In the present study, the role of IL-37 in CHD was elucidated. The mRNA expression and protein levels of IL-37 in the PBMCs of CHD patients were examined compared to those in the healthy controls (HCs). Moreover, the laboratory characteristics and the correlation between the serum levels of IL-37 and clinical manifestations of CHD were analysed. The expression levels of the pro-inflammatory cytokines IL-17, IL-6, TNF- $\alpha$, and IL-1 $\beta$ in PBMCs with or without stimulation using the recombinant IL-37 protein were detected to further explore the function of IL-37 in CHD.

\section{Materials and methods}

HCs and CHD patients. Ninety-two CHD patients admitted to Peking University Shenzhen Hospital, Shenzhen, China from March 2018 to September 2018, including thirty patients with AMI, thirty-one patients with UAP, and thirty-one patients with SAP, were enrolled for this study.

All patients were diagnosed and classified according to the clinical evidences of CHD, including their symptoms, history, electrocardiogram, echocardiography, coronary angiography, and blood tests of myocardial enzymes. The nomenclature and criteria for the diagnosis of ischemic heart disease were considered the diagnostic criteria for each patient (25). Forty-six age- and sex-matched healthy volunteers from the physical examination department of the same hospital were enrolled in this study as HCs. Participants who had autoimmune diseases, infections, malignant tumours, or other chronic inflammatory diseases were excluded from the study. Table I reveals the clinical and laboratory characteristics of the study subjects. This research was conducted with the approval of the Review Board of the Peking University Shenzhen Hospital; written informed consent was obtained from all the participants.

Blood sample collection and PBMC isolation. Fasting venous blood ( $3 \mathrm{ml}$ per subject) was obtained from the CHD patients and HCs. Blood samples were collected and analysed within $3 \mathrm{~h}$. PBMCs were isolated under sterile conditions via Ficoll-Paque Plus (TBD Science) density gradient centrifugation. The collected cells were stored at $-80^{\circ} \mathrm{C}$ before RNA extraction or before they were cultured. The serum samples were also stored at $-80^{\circ} \mathrm{C}$ before the detection of the cytokines.

Recombinant human IL-37 protein. In a previous study by our laboratory, the human recombinant IL-37 protein had been successfully cloned, described, and purified, and its functions have already been confirmed (20).

Cell culture conditions. The culture medium consisted of RPMI-1640 medium (Hyclone; Thermo Fisher Scientific, Inc.) supplemented with $100 \mathrm{IU} / \mathrm{ml}$ penicillin, $100 \mu \mathrm{g} / \mathrm{ml}$ streptomycin (Beyotime Institute of Biotechnology), and 10\% foetal calf serum (Gibco; Thermo Fisher Scientific, Inc.). Whole PBMCs were cultured at $37^{\circ} \mathrm{C}$ under a humidified atmosphere containing $5 \% \mathrm{CO}_{2}$. To determine the mRNA expression levels of the cytokines, the PBMCs from both the CHD patients and HCs were divided into two groups. The PBMC samples were left untreated or stimulated with $100 \mathrm{ng} / \mathrm{ml}$ recombinant IL-37 for $6 \mathrm{~h}$. Then, the samples were further incubated with $1 \mu \mathrm{g} / \mathrm{ml}$ LPS for $4 \mathrm{~h}$. After the extraction of the total RNAs, RT-PCR was used to analyse the transcription levels of the cytokines. To analyse the protein levels of the cytokines in the PBMCs, the cells were left untreated or treated with human recombinant IL-37 $(100 \mathrm{ng} / \mathrm{ml})$ for $24 \mathrm{~h}$, and then incubated with $1 \mu \mathrm{g} / \mathrm{ml}$ LPS for $8 \mathrm{~h}$. For the subsequent cytokine analysis using ELISA, the culture supernatants were collected and stored at $-80^{\circ} \mathrm{C}(26)$.

RNA extraction and real-time polymerase chain reaction $(R T-P C R)$. According to the manufacturer's protocol, TRIzol (Invitrogen; Thermo Fisher Scientific, Inc.) was used to extract the total RNA from the PBMCs. The purity and quantity of RNA were defined based on the absorbance of the samples on an Epoch 18 multi-volume Spectrophotometer System (BioTek Instruments, Inc.) at 260 and $280 \mathrm{~nm}$. Samples with OD260/280 ratios ranging from 1.8-2.0 were selected for the subsequent reverse transcription reaction. The RNAs were reverse-transcribed into cDNAs using the iScript ${ }^{\mathrm{TM}}$ cDNA Synthesis kit (Thermo Fisher Scientific, Inc.), following the manufacturer's instructions. RT-PCR analyses were performed using a SYBR Green PCR kit and CFX96 Real-Time PCR system (both from Bio-Rad Laboratories, Inc.). PCR primers (Shanghai Sangon Pharmaceutical Co., Ltd.) used for RT-PCR were as follows: For actin- $\beta$ sense, 5'-CCTGACTGACTACCT CATGAAGG-3' and anti-sense, 5'-CGTAGCACAGCTTCT CCTTA-3' (reverse); IL-37 sense, 5'-CGGCCCTTCATCTTT 
Table I. Clinical and laboratory characteristics of the CHD patients and healthy controls.

\begin{tabular}{|c|c|c|c|c|}
\hline Characteristics & AMI $(n=30)$ & $\operatorname{UAP}(\mathrm{n}=31)$ & $\operatorname{SAP}(n=31)$ & $\mathrm{HCs}(\mathrm{n}=46)$ \\
\hline \multicolumn{5}{|l|}{ Clinical characteristics } \\
\hline Male, n (\%) & $21(70)$ & $22(71)$ & $21(67.7)$ & $30(65.2)$ \\
\hline Age (years) & $63.27 \pm 12.18$ & $64.52 \pm 11.84$ & $67.35 \pm 11.59$ & $65 \pm 13.72$ \\
\hline $\mathrm{BMI}$ & $25.64 \pm 1.44$ & $24.95 \pm 1.41$ & $24.47 \pm 1.49$ & $23.86 \pm 1.34$ \\
\hline Hypertension, n (\%) & $19(63.3)$ & $15(48.4)$ & $16(51.6)$ & - \\
\hline Diabetes, n $(\%)$ & $4(13.3)$ & $5(16.1)$ & $2(6.4)$ & - \\
\hline Family history, n (\%) & $7(23.3)$ & $10(32.3)$ & $9(29)$ & - \\
\hline Smoking, n (\%) & $17(56.7)$ & $17(54.8)$ & $14(45.2)$ & - \\
\hline \multicolumn{5}{|c|}{ Laboratory characteristics } \\
\hline $\operatorname{LVEF}(\%)$ & $50.67 \pm 3.99$ & $56.48 \pm 2.47$ & $56.77 \pm 2.12$ & - \\
\hline $\mathrm{cTnT}(\mu \mathrm{g} / \mathrm{l})$ & $0.87 \pm 1.53$ & $0.48 \pm 1.25$ & $0.03 \pm 0.03$ & - \\
\hline NT-proBNP (pg/ml) & $824.23 \pm 149.49$ & $475.32 \pm 102.61$ & $308.9 \pm 99.27$ & - \\
\hline CHOL $(\mathrm{mmol} / \mathrm{l})$ & $4.72 \pm 0.78$ & $4.63 \pm 0.72$ & $4.52 \pm 0.76$ & $4.45 \pm 0.51$ \\
\hline $\mathrm{TG}(\mathrm{mmol} / \mathrm{l})$ & $1.87 \pm 0.5$ & $1.96 \pm 0.66$ & $1.78 \pm 0.51$ & $1.65 \pm 0.44$ \\
\hline LDL (mmol/l) & $2.87 \pm 0.59$ & $2.75 \pm 0.39$ & $2.78 \pm 0.52$ & $2.69 \pm 0.49$ \\
\hline $\operatorname{HDL}(\mathrm{mmol} / \mathrm{l})$ & $1.08 \pm 0.3$ & $1.13 \pm 0.34$ & $1.15 \pm 0.28$ & $1.27 \pm 0.34$ \\
\hline
\end{tabular}

Unless otherwise indicated, the values are expressed as the mean \pm standard deviations. There were no significant differences between CHD patients and $\mathrm{HCs}$ in terms of age and sex; The normal ranges of the clinical indexes are as follows: LVEF, $>55 \%$; cTnT, 0.02-0.13 $\mu \mathrm{g} / \mathrm{l}$; NT-proBNP, $<300 \mathrm{pg} / \mathrm{ml}$; CHOL , $<5.20 \mathrm{mmol} / \mathrm{l}$; TG, $<1.70 \mathrm{mmol} / \mathrm{l}$; LDL, $\leq 3.12 \mathrm{mmol} / \mathrm{l}$; HDL, >1.04 mmol/1. CHD, coronary heart disease; HCs, healthy controls; BMI, Body mass index; LVEF, Left ventricular ejection fraction; cTnT, cardiac troponin T; NT-proBNP, amino-N-terminal pro-plasma brain natriuretic peptide; CHOL, cholesterol; TG, triglyceride; LDL, low-density lipoprotein; HDL, high-density lipoprotein.

TATAGG-3' and anti-sense, 5'-TTTATCTGTCACCCCAAC AGG-3' (reverse); TNF- $\alpha$ sense, 5'-ACCTCTCTCTAATCA GCCCTCT' and anti-sense, 5'-GGGTTTGCTACAACATGG GCTA-3' (reverse); IL-1 $\beta$ sense, 5'-CCACAGACCTTCCAG GAGAAT-3' and anti-sense, 5'-GTGCACATAAGCCTCGTT ATCC-3' (reverse); IL-6 sense, 5'-AGCCACTCACCTCTT CAGAAC-3' and anti-sense, 5'-ACATGTCTCCTTTCTCAG GGC-3' (reverse); IL-17 sense, 5'-GTCAACCTGAACATC CATAACC-3' and anti-sense, 5'-GCACTTTGCCTCCCA GAT-3' (reverse). The relative mRNA levels of the target genes were calculated by the $2^{-\Delta \Delta \mathrm{Cq}}$ method (27), with normalisation to the mRNA expression levels of $\beta$-actin (the internal reference gene).

ELISA kits. The serum IL-37 levels were detected using a commercially available enzyme-linked immunosorbent assay (ELISA) kit (AdipoGen Life Sciences). The levels of the cytokines IL-17, IL-6, TNF- $\alpha$, and IL-1 $\beta$ in the sera and cell culture supernatants were determined using the eBioscience ELISA Kit (Thermo Fisher Scientific, Inc.), following the manufacturer's instructions.

Statistical analysis. Data were represented as the mean values [ \pm standard deviations (SDs)], and were analysed using the GraphPad Prism V.5.00 software (GraphPad Software, Inc.). Differences between two groups were analysed using the Mann-Whitney $U$-test for nonparametric data, while the differences between the samples left unstimulated or those stimulated with human recombinant IL-37 were analysed using the Wilcoxon rank sum test. The association between the serum IL-37 levels and clinical indexes, as well as the serum cytokine levels, was calculated by performing Spearman's correlation test. For all experiments, $\mathrm{P}<0.05$ was considered to indicate a statistically significant difference.

\section{Results}

Increased expression of IL-37 mRNA in PBMCs from CHD patients, especially AMI and UAP patients. To study the correlation between CHD and the IL-37 levels, RT-PCR was performed to detect the IL-37 mRNA expression in PBMCs from the CHD patients and HCs. The results indicated that the expression of IL-37 mRNA in the PBMCs from the HCs was lower than that in the PBMCs from the CHD patients (Fig. 1A). Then, the IL-37 mRNA expression levels in the PBMCs from the different CHD patients, such as AMI, UAP, and SAP patients, were analysed. Compared to the SAP patients, a marked increase of the IL-37 mRNA levels was observed in the AMI and UAP patients, while no significant difference between the IL-37 mRNA levels of the AMI and UAP patients was observed. However, compared with the HCs, there was a significant upregulation of the IL-37 mRNA expression in the AMI and UAP patients, while no difference between the IL-37 mRNA levels of the HCs and SAP patients was observed (Fig. 1A).

Increased serum IL-37 levels in CHD patients, especially AMI and UAP patients. As revealed in Fig. 1B, the serum IL-37 protein levels were notably lower in the HCs than in the CHD patients. Subsequently, the CHD patients were divided into 

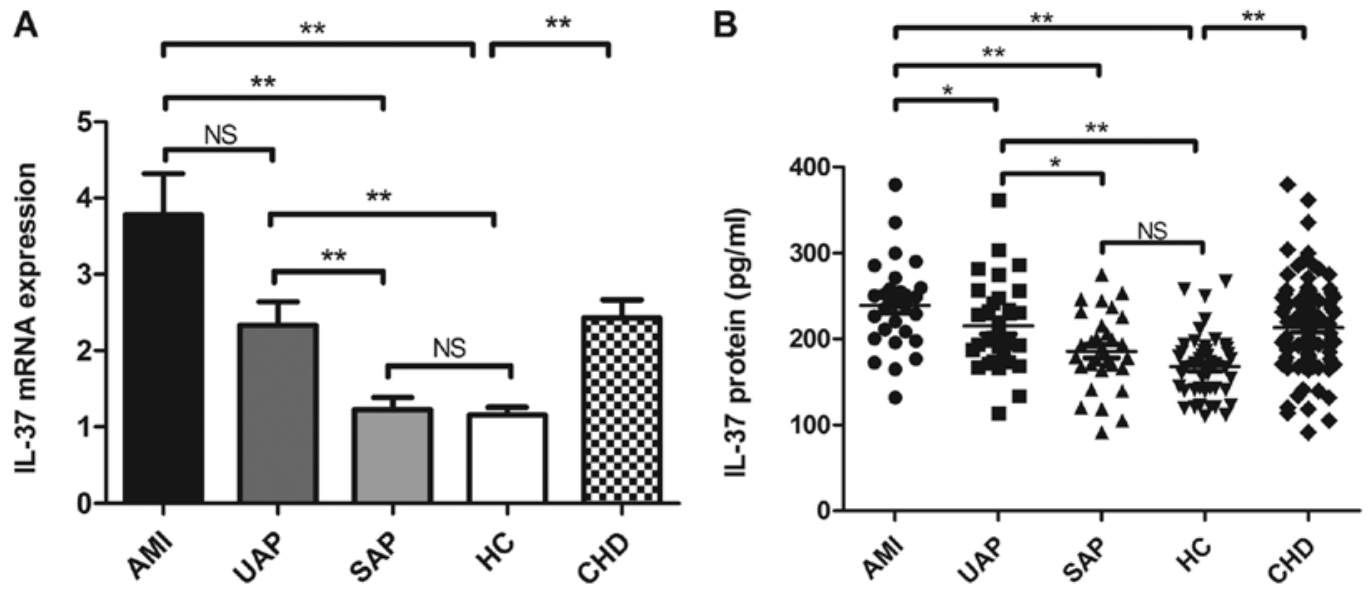

Figure 1. Comparison of the mRNA and protein levels of IL-37 between the CHD patients and HCs. (A) The IL-37 mRNA expression levels in PBMCs from AMI $(n=30)$, UAP $(n=31)$, and SAP patients $(n=31)$, and HCs $(n=46)$ were assessed by RT-PCR; the results are expressed as the means \pm SDs. (B) Serum IL-37 levels in AMI $(\mathrm{n}=30)$, UAP $(\mathrm{n}=31)$, and SAP patients $(\mathrm{n}=31)$, and HCs $(\mathrm{n}=46)$ were measured by ELISA. Each symbol represents an individual CHD patient and HC. The horizontal lines indicate the median values. Differences between two groups were analysed by performing the Mann-Whitney U test for nonparametric data. " $\mathrm{P}<0.05 ;{ }^{* *} \mathrm{P}<0.01$. IL-37, interleukin-37; CHD, coronary heart disease; PBMCs, peripheral blood mononuclear cells; AMI, acute myocardial infarction; UAP, unstable angina pectoris; SAP, stable angina pectoris; HC, healthy control; NS, not significant.
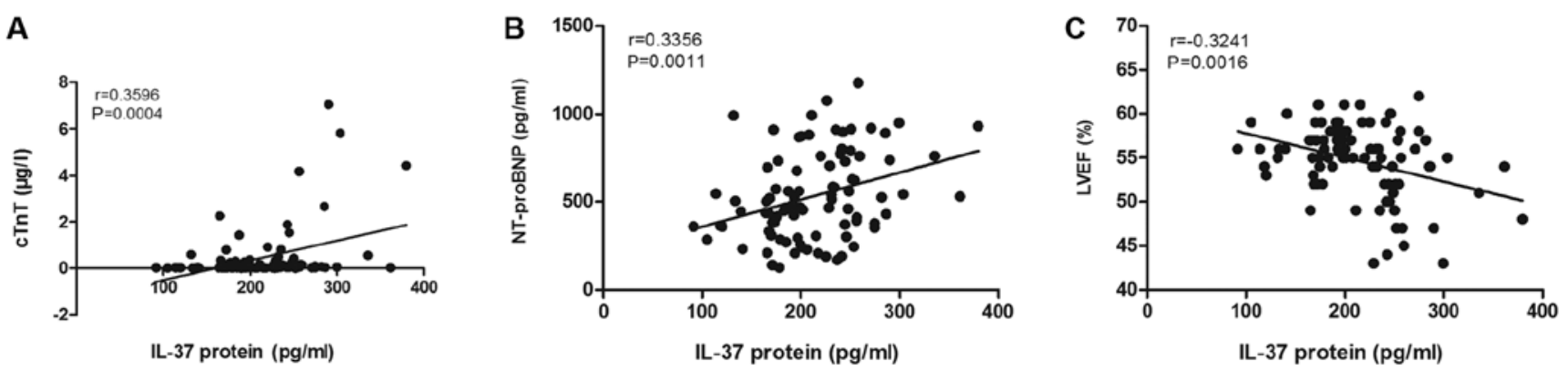

Figure 2. Correlation of the serum IL-37 levels and the clinical indexes. The serum IL-37 levels in the CHD patients were positively correlated with the (A) cTnT and (B) NT-proBNP levels and negatively correlated with the (C) LVEF. Each symbol represents an individual patient. The correlations were evaluated by performing Spearman's non-parametric test. $\mathrm{P}<0.05$, indicated a statistically significant difference. IL-37, interleukin-37; CHD, coronary heart disease; cTnT, cardiac troponin T; NT-proBNP, amino-N-terminal pro-plasma brain natriuretic peptide; LVEF, left ventricular ejection fraction.

different groups, including the AMI, UAP, and SAP groups to analyse the data. The serum IL-37 protein levels revealed the following trend: AMI group > UAP group > SAP group, which was consistent with the disease severity observed in the cases of the patients from each group. Furthermore, compared to the HCs, a significant increase of the serum IL-37 levels was observed in the AMI and UAP patients, while no significant difference between the serum IL-37 levels of the SAP patients and HCs was observed (Fig. 1B).

Association between the serum IL-37 levels and clinical indexes in CHD patients. Subsequently, the latent association of the IL-37 levels with the clinical indexes, including the left ventricular ejection fraction (LVEF), amino-N-terminal pro-plasma brain natriuretic peptide (NT-proBNP) levels, and cardiac troponin $\mathrm{T}$ (cTnT) levels, was analysed. The results indicated that the serum IL-37 levels were positively associated with the NT-proBNP (Fig. 2B, r=0.3356, P=0.0011) and cTnT levels (Fig. 2A; r=0.3596, $\mathrm{P}=0.0004$ ). In addition, there was a significant negative association between the serum IL-37 levels and the LVEF (Fig. 2C, r=0.3241, P=0.0016). However, there was no correlation between the serum IL-37 levels and the triglyceride (TG), cholesterol (CHOL), high-density lipoprotein (HDL), or low-density lipoprotein (LDL) levels (Table II).

The levels of pro-inflammatory cytokines are higher in CHD patients. Previously published studies have revealed that IL-17, IL-6, TNF- $\alpha$, and IL-1 $\beta$ have a major effect on the nosogenesis of CHD (9-19). The results obtained in the present study were consistent with this finding. As anticipated, the serum levels of IL-17, IL-6, TNF- $\alpha$, and IL-1 $\beta$ were significantly higher in the CHD patients than in the HCs (Fig. 3A-D).

Correlation between the serum IL-37 and pro-inflammatory cytokine levels. Spearman's correlation test was performed to explore the correlation between the IL-37 levels and IL-17, IL-6, TNF- $\alpha$, and IL-1 $\beta$ levels in CHD patients. The results indicated that the serum IL-37 levels were positively associated with the serum levels of TNF- $\alpha$ (Fig. 4A, $r=0.3215$, $\mathrm{P}=0.0018$ ), IL-6 (Fig. 4C, $\mathrm{r}=0.2412, \mathrm{P}=0.0206$ ), and IL-17 (Fig. 4D, $r=0.2537, \mathrm{P}=0.0147$ ). However, there was no significant correlation between the serum levels of IL-37 and IL-1 $\beta$ (Fig. 4B, r=0.1288, $\mathrm{P}=0.2209$ ) in the CHD patients. 
Table II. Correlation between the IL-37 levels and the pro-inflammatory cytokines levels as well as the clinical indexes for CHD.

\begin{tabular}{lcc}
\hline Parameters & Correlation coefficient $(r)$ & P-value \\
\hline TNF- $\alpha$ & 0.3215 & 0.0018 \\
IL-1 $\beta$ & 0.1288 & 0.2209 \\
IL-6 & 0.2412 & 0.0206 \\
IL-17 & 0.2537 & 0.0147 \\
CHOL & 0.08379 & 0.4271 \\
TG & 0.1123 & 0.2864 \\
LDL & 0.04179 & 0.6924 \\
HDL & 0.01715 & 0.8711 \\
\hline
\end{tabular}

The correlations were evaluated by performing Spearman's non-parametric test. $\mathrm{P}<0.05$ represents a significant difference. IL-37, interleukin-37; CHD, coronary heart disease; $\mathrm{CHOL}$, cholesterol TG, triglyceride; LDL, low-density lipoprotein; HDL, high-density lipoprotein.

Recombinant IL-37 decreases the expression of pro-inflammatory cytokines in the PBMCs of CHD patients. It has been reported that IL-37 exerts an anti-inflammatory effect on inflammatory and auto-immune diseases through the downregulation of inflammatory responses $(20,28,29)$. To verify whether IL-37 can reduce the expression levels of pro-inflammatory cytokines during CHD, a series of tests were performed. First, the recombinant human IL-37 protein was expressed and purified via previously described methods (20). Next, the PBMCs isolated from the CHD patients and HCs were left untreated or stimulated with recombinant IL-37, and then stimulated with LPS. The culture supernatants and cells were collected for the RT-PCR and ELISA analysis, respectively. It was concluded that the mRNA expression levels of TNF- $\alpha$ (Fig. 5A), IL-1 $\beta$ (Fig. 5B), IL-6 (Fig. 5C), and IL-17 (Fig. 5D) in the PBMCs of CHD patients were significantly reduced by IL-37 treatment. Additionally, IL-37 treatment significantly decreased the secreted amounts of TNF- $\alpha$ (Fig. 5E), IL-1 $\beta$ (Fig. 5F), IL-6 (Fig. 5G), and IL-17 (Fig. 5H) in the culture supernatants of the PBMCs from the CHD patients. However, the mRNA and protein levels of these pro-inflammatory cytokines in the PBMCs from the HCs exhibited no significant changes after IL-37 stimulation (Fig. 5A-H).

\section{Discussion}

Recently, IL-37 has been widely discussed as a cytokine with anti-inflammatory effects in various diseases, including autoimmune diseases, infectious diseases, metabolic diseases, and ischemia-reperfusion injury (20-22,28-32). Referring to coronary heart events, plasma IL-37 concentrations were increased in patients with coronary artery calcification, indicating that IL-37 may be a predictor of CHD $(24,33)$. A previous animal experiment indicated that IL-37 could attenuate inflammatory response and thus protected cardiac function in old endotoxemia mice (25). In addition, our previous studies have confirmed that IL-37, an immune mediator, can restrain the inflammatory responses associated with autoimmune diseases such as ankylosing spondylitis (AS), SLE, and Graves' disease (GD) $(20,26,34)$. However, how IL-37 is involved in heart diseases, especially CHD, is still unknown.

Although the understanding of the pathological mechanism in CHD is limited, previously published research has indicated that CHD is a chronic inflammatory disease, based on the observation of atherosclerosis. Numerous pro-inflammatory cells and cytokines have important effects on the nosogenesis of CHD. Previous studies have demonstrated that mononuclear macrophages, especially PBMCs, are closely related to the development of CHD $(8,35)$. After long-term dyslipidaemia, monocytes from PBMCs will enter the intima and transform into macrophages. After engulfing the lipids, these macrophages transform into foam cells, thus forming the early atherosclerotic plaques; thus, they play an important role in acute CHD. Boraschi et al have revealed that IL-37 is highly expressed in human coronary atherosclerotic plaque foam cells, indicating that IL-37 is also involved in the process of CHD (21). In the present study, it was demonstrated that IL-37 is involved in CHD by revealing that the IL-37 mRNA expression and the serum IL-37 levels were significantly higher in CHD patients than in the HCs.

In addition, the CHD patients were divided into AMI, UAP, and SAP groups according to the CHD disease activity; it was revealed that the serum IL-37 protein levels were associated with the severity of clinical symptoms in the CHD patients (based on the trend of the IL-37 levels: AMI group > UAP group $>$ SAP group). Furthermore, the present data revealed the correlation between the levels of IL-37 and several useful markers of the CHD disease activity, such as the cTnT levels, NT-proBNP levels, and LVEF. Clinically, increased cTnT levels indicate myocardial ischemia, while elevated NT-proBNP levels and decreased LVEF values indicate cardiac insufficiency. In most instances, the more abnormal the levels of the aforementioned indicators, the more active and severe the CHD. Thus, these results also indicated that IL-37 was correlated with the activity and severity of CHD.

A number of cytokines, including TNF- $\alpha$, IL-17, IL-6, and IL-1 $\beta$, have been detected in the development of CHD (9-19). In the present study, increased serum levels of TNF- $\alpha$, IL-17, IL- 6 , and IL- $1 \beta$ were also observed in CHD patients, compared with the HCs. Notably, the IL-37 levels were positively associated with the levels of these major pro-inflammatory cytokines in CHD patients. However, there was no significant difference among the subgroups of CHD (AMI, UAP, and SAP groups). These results were consistent with the findings of our prior research on autoimmune diseases such as AS, SLE, and GD $(20,26,34)$, which revealed that pro-inflammatory cytokines were positively correlated with IL-37 production in inflammatory response. As for why there was no significance among subgroups of CHD, it is surmised that the sample size should be larger and thus differences in each subgroup may be exhibited between groups. In addition, the classification of AMI, UAP and SAP is according to clinical evidence of CHD, including their symptoms, history, electrocardiogram, echocardiography, coronary angiography, and blood tests of myocardial enzymes. These three subgroups are not strictly classified according to the course of disease onset and thus 
A

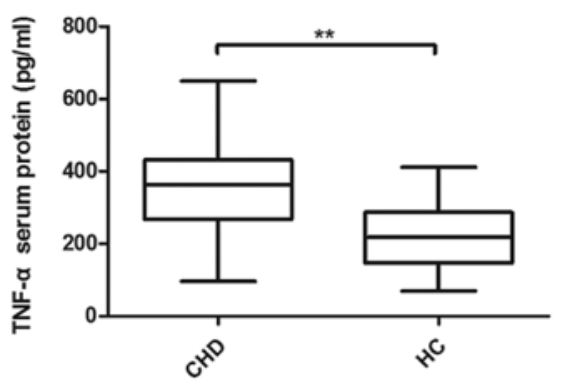

C

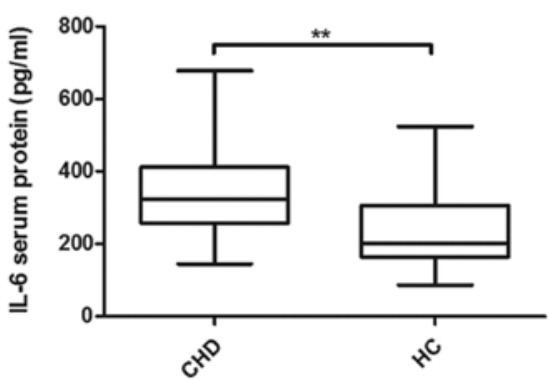

B

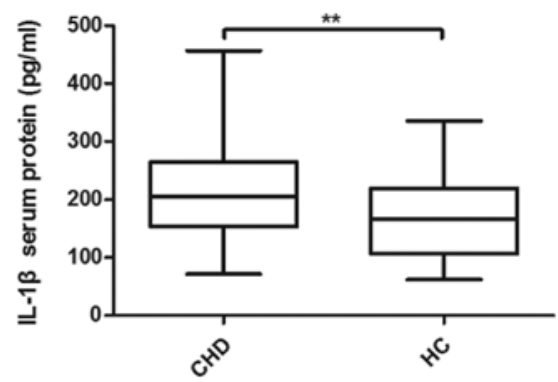

D

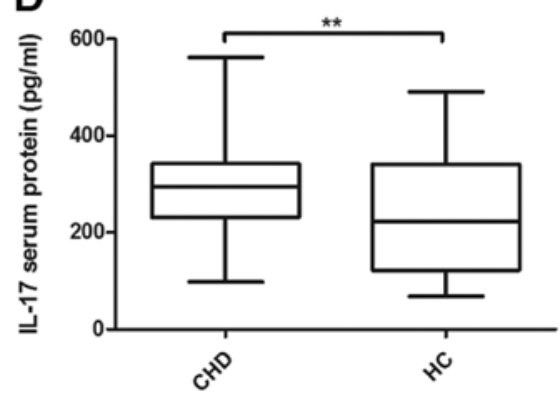

Figure 3. Comparison of the serum protein levels of the inflammatory cytokines among the CHD patients and HCs. Serum levels of the cytokines (A) TNF- $\alpha$, (B) IL-1 $\beta$, (C) IL-6, and (D) IL-17 in the CHD patients $(n=92)$ and HCs $(n=46)$ were analysed by ELISA. The results are depicted as box plots, with the medians (horizontal lines within each box) and 10, 25, 75, and 90th percentiles (bottom bar, bottom of the box, top of the box, and top bar, respectively). The differences between the CHDs and HCs were analysed by performing the Mann-Whitney $\mathrm{U}$ test. ${ }^{* *} \mathrm{P}<0.01$, indicated a statistically significant difference. CHD, coronary heart disease; HCs, healthy controls.
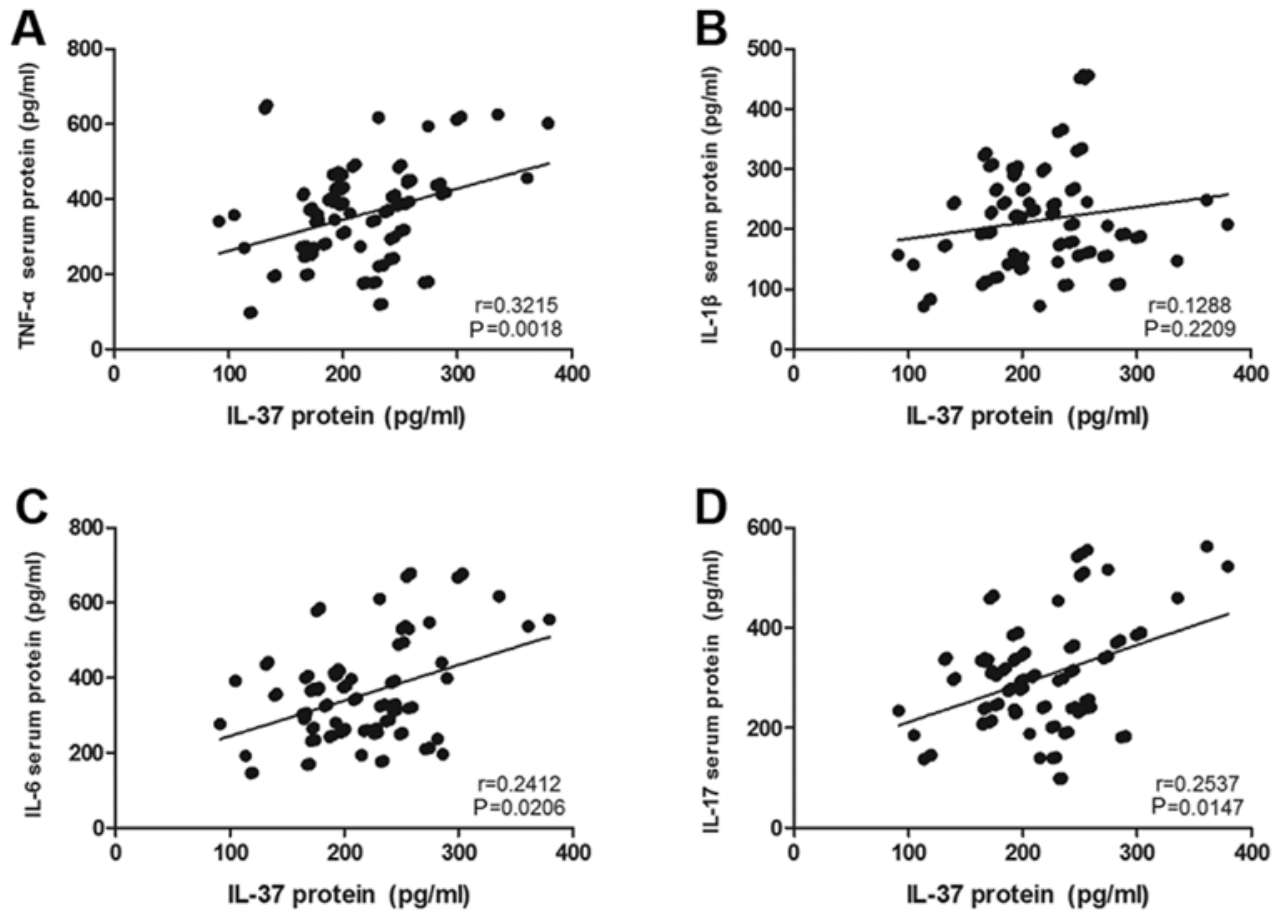

Figure 4. Correlation between the serum levels of IL-37 and pro-inflammatory cytokines in CHD patients. Serum IL-37 levels were positively correlated with the levels of (A) TNF- $\alpha$, (C) IL-6, and (D) IL-17, but were independent of the levels of (B) IL-1 $\beta$. Each symbol represents an individual patient. The correlations were evaluated by performing the Spearman's non-parametric test. NS, not significant. IL-37, interleukin-37; CHD, coronary heart disease.

the levels of inflammatory factors are not necessarily different in each group. In summary, the results of the present study indicated that the inflammatory reactions associated with CHD and the related pro-inflammatory cytokines are probably involved in a positive feedback to induce the expression of IL-37 during CHD-related complications. Moreover, it has been revealed that IL-37 levels can be induced by TNF- $\alpha$ through the activator protein (AP)-1 signalling pathway and activation 

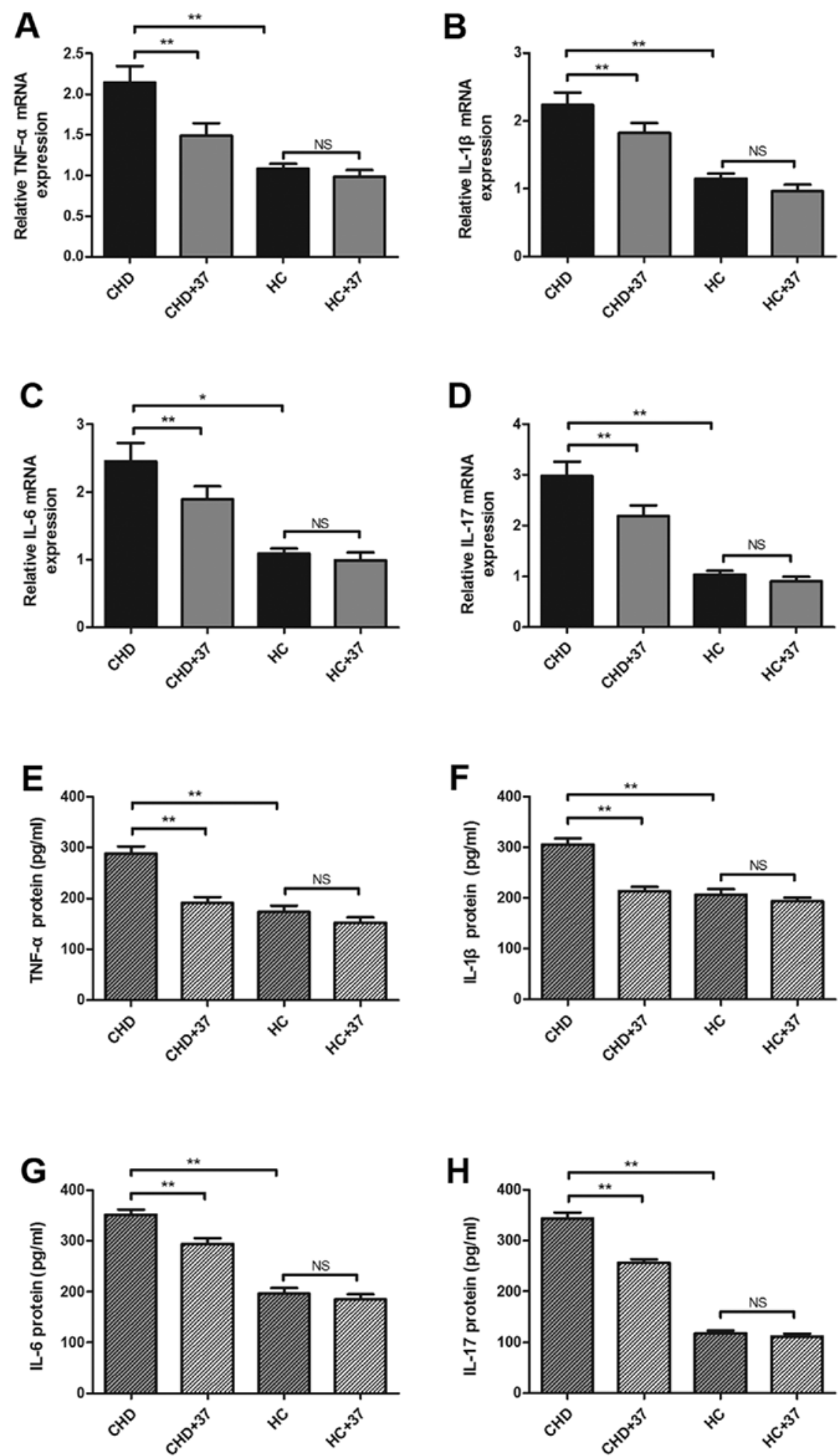

Figure 5. Recombinant IL-37 decreases the expression of pro-inflammatory cytokines in the PBMCs of CHD patients. PBMCs from CHD patients (n=92) and HCs $(\mathrm{n}=46)$ were left untreated or stimulated with IL-37 $(100 \mathrm{ng} / \mathrm{ml})$ for $6 \mathrm{~h}$, and incubated with LPS $(1 \mu \mathrm{g} / \mathrm{ml})$ for $4 \mathrm{~h}$; the total RNAs were then extracted and the mRNA expression levels of (A) TNF- $\alpha$, (B) IL-1 $\beta$, (C) IL-6, and (D) IL-17 were analysed by RT-PCR. The PBMCs from CHD patients (n=92) and HCs $(\mathrm{n}=46)$ were left untreated or stimulated with IL-37 $(100 \mathrm{ng} / \mathrm{ml})$ for $24 \mathrm{~h}$, and incubated with LPS $(1 \mu \mathrm{g} / \mathrm{ml})$ for $8 \mathrm{~h}$; then, the protein levels of (E) TNF- $\alpha$, (F) IL-1 $\beta$, (G) IL-6, and (H) IL-17 in the supernatants of the cell cultures were examined by ELISA. The results are expressed as the means \pm SDs; P $<0.05$ represents a significant difference. CHD + IL-37, PBMCs from CHD patients stimulated with the recombinant IL-37; HC + IL-37, PBMCs from HCs stimulated with the recombinant IL-37; ${ }^{*} \mathrm{P}<0.05$ represents a significant difference; ${ }^{* *} \mathrm{P}<0.01$ represents a significant difference. IL-37, interleukin-37; PBMCs, peripheral blood mononuclear cells; CHD, coronary heart disease; HCs, healthy controls; NS, not significant.

of nuclear factor (NF)-kB in intestinal epithelial cells (36). In an in vitro study, Nold et al have also demonstrated that TNF- $\alpha$ induces IL-37 expression in the PBMCs of HCs (22), indicating that TNF- $\alpha$ is one of the inducers involved in the upregulation of IL-37 during CHD. Moreover, in previous clinical trials, anti-TNF- $\alpha$ antibodies have been revealed to successfully block the Th17 and IL-6 responses, revealing that IL-6 and other cytokines are important downstream effectors 
of the TNF- $\alpha$ pathway $(37,38)$. In another study, siRNA knockdown of IL-37 in PBMCs increased the production of IL-6 and TNF- $\alpha$ induced by inflammatory stimuli (39). Therefore, it is speculated that the inflammatory signals resulting from the TNF- $\alpha$ pathway induce the expression of the cytokine IL-37, which serves as an inhibitor of excessive inflammation, and thus attenuates the inflammatory response in CHD. Further studies are required to clearly determine the role of the TNF- $\alpha$ pathway in regulating the expression of IL-37.

The aforementioned results could be classified into 3 types of parameters that are closely related to CHD: Pro-inflammatory cytokines (IL-17, IL-6, TNF- $\alpha$ and IL-1 $\beta$ ), variables related to cardiac function clinically (cTnT, NT-proBNP and LVEF) as well as biochemical variables related to lipid profiles (cholesterol, triglyceride, low-density lipoprotein and high-density lipoprotein). These parameters contain the laboratory findings and clinic assessments, which are commonly used in the study of cardiovascular diseases. However, it is unknown whether IL-37 reflects arterial calcification or not. Additionally, the area of arterial plaques is the most frequently used index reflecting the degree of atherosclerosis of CHD. Since it is difficult to assess the arterial plaque areas of all patients, an animal experiment is required to determine whether IL-37 can delay the formation of arterial plaque. In a future study, animal experiments will be performed to assess the area of arterial plaque and serum calcium level as well as the level of osteoprotegerin which may reflect arterial calcification. These experiments can further investigate whether IL-37 can protect against the process of arterial calcification and formation of arterial plaque in CHD.

Recently, IL-37 was revealed to reduce pro-inflammatory cytokine production in inflammatory diseases, acting as a negative regulator of inflammation $(20,26,34,40)$. To further analyse the effects of IL-37, PBMCs from CHD patients and HCs were stimulated using recombinant human IL-37. In the present study, treatment with recombinant IL-37 significantly decreased the expression of IL-17, IL-6, TNF- $\alpha$, and IL-1 $\beta$ in LPS-stimulated PBMCs from the CHD patients, but no such decrease in the levels of these pro-inflammatory cytokines was observed in the LPS-stimulated PBMCs from the HCs. Despite the elusive inhibition of pro-inflammatory cytokine-related signalling pathways by IL-37, Nold et al have reported that the expression of STAT3 can be decreased by IL-37 (22); STAT3 has been reported to be closely associated with CHD $(41,42)$. In addition, IL-37 can form functional complexes with Smad3 (a mothers against decapentaplegic related-protein) to inhibit the activity of signaling molecules STAT3 by reducing the phosphorylation of STAT3, which is a key player in the signaling transduction of pro-inflammatory cytokines $(43,44)$. Thus, it is inferred that IL-37 may attenuate the production of pro-inflammatory cytokines by inhibiting the phosphorylation of STAT3, in order to reduce the inflammatory reactions associated with CHD. However, it is uncertain whether IL-37 could directly inhibit phosphorylation of STAT3 or reduce upstream kinases indirectly.

These findings provide a new perspective for addressing the balance between the levels of pro-inflammatory cytokines and IL-37 in cardiovascular diseases such as CHD. However, further research on the mechanisms underlying the function of IL-37 in the pathogenesis of CHD is required in the future.
In summary, the present results indicated that the expression of the pro-inflammatory cytokines IL-17, IL-6, TNF- $\alpha$, and IL- $1 \beta$ in CHD patients was promoted due to the activation of the inflammatory response. Furthermore, the levels of IL-37 were increased in CHD patients, and they are associated with the expression of pro-inflammatory cytokines, disease activity, and certain clinical indexes. This study proposed that in the presence of the inflammatory response associated with CHD, both pro-inflammatory and anti-inflammatory activities are activated concurrently.

Increased levels of IL-37 were positively associated with pro-inflammatory cytokines (IL-17, IL-6, and TNF- $\alpha$ ) in patients with CHD, resulting in the efficient alleviation of the expression of these pro-inflammatory cytokines. This finding indicates that IL-37 can restrain excessive inflammation during CHD. It maintains the balance of the immune response by mitigating the expression of pro-inflammatory factors. Thus, it exerts an anti-inflammatory effect in CHD. The present research indicated that IL-37 may be used as a clinical indicator to monitor the condition of CHD patients, guide their treatment, or determine their prognosis in the future. In addition, this study offers a new potential target (IL-37) for CHD therapy. The association between IL-37 and CHD will further be studied to explore new research directions for the prevention and treatment of CHD.

\section{Acknowledgements}

Not applicable.

\section{Funding}

The present study was supported bya grant from the Shenzhen Science and Technology Innovation Committee Foundation (grant no. JCYJ20160422144926325).

\section{Availability of data and materials}

The datasets used and analyzed during the current study are available from the corresponding author on reasonable request.

\section{Authors' contributions}

CS and HL contributed to the conception and design of the study, data acquisition, data analysis and manuscript writing. HL and BC participated in critical revision of the manuscript. CS, HL and BC participated in data collection, interpretation of cell culture, RT-PCR and ELISA experiments. BP, WW, FC and XD participated in the statistical analysis and manuscript writing. JD participated in the conception and design of the study as well as provided patient samples, and clinical and laboratory data. $\mathrm{ZH}$ and $\mathrm{CY}$ conceived the experiments, analyzed the data, read and critically revised the manuscript. All authors read and gave final approval of the version to be published.

\section{Ethics approval and consent to participate}

This research was conducted with the approval of the Review Board of the Peking University Shenzhen Hospital; written informed consent was obtained from all the participants. 


\section{Patient consent for publication}

Not applicable.

\section{Competing interests}

The authors declare that they have no competing interests.

\section{References}

1. Wong ND: Epidemiological studies of CHD and the evolution of preventive cardiology. Nat Rev Cardiol 11: 276-289, 2014.

2. Libby P and Theroux P: Pathophysiology of coronary artery disease. Circulation 111: 3481-3488, 2005.

3. Ridker PM and Lüscher TF: Anti-inflammatory therapies for cardiovascular disease. Eur Heart J 35: 1782-1791, 2014.

4. Boisvert WA: The participation of inflammatory cells in atherosclerosis. Drugs Today (Barc) 37: 173-179, 2001.

5. Libby P: Inflammation in atherosclerosis. Arterioscler Thromb Vasc Biol 32: 2045-2051, 2012

6. Seneviratne AN and Monaco C: Role of inflammatory cells and toll-like receptors in atherosclerosis. Curr Vasc Pharmacol 13: 146-160, 2015

7. Chistiakov DA, Orekhov AN and Bobryshev YV: Immuneinflammatory responses in atherosclerosis: Role of an adaptive immunity mainly driven by $\mathrm{T}$ and $\mathrm{B}$ cells. Immunobiology 221 : 1014-1033, 2016.

8. Martinet W, Schrijvers DM and De Meyer GR: Molecular and cellular mechanisms of macrophage survival in atherosclerosis. Basic Res Cardiol 107: 297, 2012.

9. Bruunsgaard H, Skinhøj P, Pedersen AN, Schroll M and Pedersen BK: Ageing, tumour necrosis factor-alpha (TNF-alpha) and atherosclerosis. Clin Exp Immunol 121: 255-260, 2000.

10. Popa C, Netea MG, van Riel PL, van der Meer JW and Stalenhoef AF: The role of TNF-alpha in chronic inflammatory conditions, intermediary metabolism, and cardiovascular risk. J Lipid Res 48: 751-762, 2007.

11. Sbarsi I, Falcone C, Boiocchi C, Campo I, Zorzetto M, De Silvestri A and Cuccia M: Inflammation and atherosclerosis: The role of TNF and TNF receptors polymorphisms in coronary artery disease. Int J Immunopathol Pharmacol 20: 145-154, 2007.

12. Zhang H, Park Y, Wu J, Chen XP, Lee S, Yang J, Dellsperger KC and Zhang C: Role of TNF-alpha in vascular dysfunction. Clin Sci (Lond) 116: 219-230, 2009.

13. Iida S and Komiyama N: Balance between interleukin-1beta and interleukin-1 receptor antagonist in the development of atherosclerosis. A polymorphism in the interleukin-1 receptor antagonist. Circ J 73: 1401-1402, 2009.

14. Lin SJ, Yen HT, Chen YH, Ku HH, Lin FY and Chen YL: Expression of interleukin-1 beta and interleukin-1 receptor antagonist in oxLDL-treated human aortic smooth muscle cells and in the neointima of cholesterol-fed endothelia-denuded rabbits. J Cell Biochem 88: 836-847, 2003.

15. Legendre F, Bogdanowicz P, Boumediene K and Pujol JP: Role of interleukin 6 (IL-6)/IL-6R-induced signal tranducers and activators of transcription and mitogen-activated protein kinase/extracellular. J Rheumatol 32: 1307-1316, 2005.

16. Biasucci LM, Liuzzo G, Fantuzzi G, Caligiuri G, Rebuzzi AG, Ginnetti F, Dinarello CA and Maseri A: Increasing levels of interleukin (IL)-1Ra and IL-6 during the first 2 days of hospitalization in unstable angina are associated with increased risk of in-hospital coronary events. Circulation 99: 2079-2084, 1999.

17. Kotla S, Singh NK, Heckle MR, Tigyi GJ and Rao GN: The transcription factor CREB enhances interleukin-17A production and inflammation in a mouse model of atherosclerosis. Sci Signal 6: ra83, 2013.

18. Usui F, Kimura H, Ohshiro T, Tatsumi K, Kawashima A, Nishiyama A, Iwakura Y, Ishibashi $\mathrm{S}$ and Takahashi $\mathrm{M}$ : Interleukin-17 deficiency reduced vascular inflammation and development of atherosclerosis in Western diet-induced apoE-deficient mice. Biochem Biophys Res Commun 420: 72-77, 2012.

19. Zhu F, Wang Q, Guo C, Wang X, Cao X, Shi Y, Gao F, Ma C and Zhang L: IL-17 induces apoptosis of vascular endothelial cells: A potential mechanism for human acute coronary syndrome. Clin Immunol 141: 152-160, 2011.
20. Ye L, Ji L, Wen Z, Zhou Y, Hu D, Li Y, Yu T, Chen B, Zhang J, Ding L, et al: IL-37 inhibits the production of inflammatory cytokines in peripheral blood mononuclear cells of patients with systemic lupus erythematosus: Its correlation with disease activity. J Transl Med 12: 69, 2014.

21. Boraschi D, Lucchesi D, Hainzl S, Leitner M, Maier E, Mangelberger D, Oostingh GJ, Pfaller T, Pixner C, Posselt G, et al: IL-37: A new anti-inflammatory cytokine of the IL-1 family. Eur Cytokine Netw 22: 127-147, 2011.

22. Nold MF, Nold-Petry CA, Zepp JA, Palmer BE, Bufler P and Dinarello CA: IL-37 is a fundamental inhibitor of innate immunity. Nat Immunol 11: 1014-1022, 2010.

23. Yu K, Min X, Lin Y, Huang Y, Huang S, Liu L, Peng Y, Meng K, Li D, Ji Q and Zeng Q: Increased IL-37 concentrations in patients with arterial calcification. Clin Chim Acta 461: 19-24, 2016

24. Li J, Zhai Y, Ao L, Hui H, Fullerton DA, Dinarello CA and Meng X: Interleukin-37 suppresses the inflammatory response to protect cardiac function in old endotoxemic mice. Cytokine 95: 55-63, 2017.

25. Nomenclature and criteria for diagnosis of ischemic heart disease. Report of the Joint International Society and Federation of Cardiology/World Health Organization task force on standardization of clinical nomenclature. Circulation 59: 607-609, 1979.

26. Chen B, Huang K, Ye L, Li Y, Zhang J, Zhang J, Fan X, Liu X, $\mathrm{Li} \mathrm{L}$, Sun $\mathrm{J}$, et al: Interleukin-37 is increased in ankylosing spondylitis patients and associated with disease activity. J Transl Med 13: 36, 2015.

27. Livak KJ and Schmittgen TD: Analysis of relative gene expression data using real-time quantitative PCR and the 2(-Delta Delta C(T)) method. Methods 25: 402-408, 2001.

28. Imaeda H, Takahashi K, Fujimoto T, Kasumi E, Ban H, Bamba S, Sonoda H, Shimizu T, Fujiyama Y and Andoh A: Epithelial expression of interleukin-37b in inflammatory bowel disease. Clin Exp Immunol 172: 410-416, 2013.

29. Zhao PW, Jiang WG, Wang L, Jiang ZY, Shan YX and Jiang YF: Plasma levels of IL-37 and correlation with TNF-alpha, IL-17A, and disease activity during DMARD treatment of rheumatoid arthritis. PLoS One 9: e95346, 2014.

30. Ballak DB, van Diepen JA, Moschen AR, Jansen HJ, Hijmans A, Groenhof GJ, Leenders F, Bufler P, Boekschoten MV, Müller M, et al: IL-37 protects against obesity-induced inflammation and insulin resistance. Nat Commun 5: 4711, 2014.

31. Moschen AR, Molnar C, Enrich B, Geiger S, Ebenbichler CF and Tilg H: Adipose and liver expression of interleukin (IL)-1 family members in morbid obesity and effects of weight loss. Mol Med 17: 840-845, 2011.

32. Sakai N, Van Sweringen HL, Belizaire RM, Quillin RC, Schuster R, Blanchard J, Burns JM, Tevar AD, Edwards MJ and Lentsch AB: Interleukin-37 reduces liver inflammatory injury via effects on hepatocytes and non-parenchymal cells. J Gastroenterol Hepatol 27: 1609-1616, 2012.

33. Chai M, Zhang HT, Zhou YJ, Ji QW, Yang Q, Liu YY, Zhao YX, Shi DM, Liu W, Yang LX, et al: Elevated IL-37 levels in the plasma of patients with severe coronary artery calcification. J Geriatr Cardiol 14: 285-291, 2017.

34. Li Y, Wang Z, Yu T, Chen B, Zhang J, Huang K and Huang Z: Increased expression of IL-37 in patients with Graves' disease and its contribution to suppression of proinflammatory cytokines production in peripheral blood mononuclear cells. PLoS One 9: e107183, 2014.

35. Swirski FK and Nahrendorf M: Leukocyte behavior in atherosclerosis, myocardial infarction, and heart failure. Science 339: 161-166, 2013.

36. Wu B, Meng K, Ji Q, Cheng M, Yu K, Zhao X, Tony H, Liu Y, Zhou Y, Chang C, et al: Interleukin-37 ameliorates myocardial ischaemia/reperfusion injury in mice. Clin Exp Immunol 176: 438-451, 2014

37. Visvanathan S, Wagner C, Marini JC, Baker D, Gathany T, Han J, van der Heijde D and Braun J: Inflammatory biomarkers, disease activity and spinal disease measures in patients with ankylosing spondylitis after treatment with infliximab. Ann Rheum Dis 67: 511-517, 2008.

38. Zaba LC, Cardinale I, Gilleaudeau P, Sullivan-Whalen M, Suárez-Fariñas M, Fuentes-Duculan J, Novitskaya I, Khatcherian A, Bluth MJ, Lowes MA and Krueger JG: Amelioration of epidermal hyperplasia by TNF inhibition is associated with reduced Th17 responses. J Exp Med 204: $3183-3194,2007$. 
39. Yang Y, Zhang ZX, Lian D, Haig A, Bhattacharjee RN and Jevnikar AM: IL-37 inhibits IL-18-induced tubular epithelial cell expression of pro-inflammatory cytokines and renal ischemia-reperfusion injury. Kidney Int 87: 396-408, 2015.

40. McNamee EN, Masterson JC, Jedlicka P, McManus M, Grenz A, Collins CB, Nold MF, Nold-Petry C, Bufler P, Dinarello CA and Rivera-Nieves J: Interleukin 37 expression protects mice from colitis. Proc Natl Acad Sci USA 108: 16711-16716, 2011.

41. Katta S, Karnewar S, Panuganti D, Jerald MK, Sastry BKS and Kotamraju S: Mitochondria-targeted esculetin inhibits PAI-1 levels by modulating STAT3 activation and miR-19b via SIRT3: Role in acute coronary artery syndrome. J Cell Physiol 233: 214-225, 2018.

42. Zheng Y, Wang Z, Deng L, Zhang G, Yuan X, Huang L, Xu W and Shen L: Modulation of STAT3 and STAT5 activity rectifies the imbalance of Th17 and Treg cells in patients with acute coronary syndrome. Clin Immunol 157: 65-77, 2015.
43. Tete S, Tripodi D, Rosati M, Conti F, Maccauro G, Saggini A Cianchetti E, Caraffa A, Antinolfi P, Toniato E, et al: IL-37 (IL-1F7) the newest anti-inflammatory cy tokine which suppresses immune responses and inflammation. Int $\mathbf{J}$ Immunopathol Pharmacol 25: 31-38, 2012.

44. Akdis M, Burgler S, Crameri R, Eiwegger T, Fujita H, Gomez E, Klunker S, Meyer N, O'Mahony L, Palomares O, et al: Interleukins, from 1 to 37, and interferon- $\gamma$ : Receptors, functions, and roles in diseases. J Allergy Clin Immunol 127: 701-721.e1-e70, 2011.

This work is licensed under a Creative Commons Attribution-NonCommercial-NoDerivatives 4.0 International (CC BY-NC-ND 4.0) License. 